\title{
Alarm- und Einsatzplanung im Krankenhaus: Vorbereitung auf Großschadenslagen
}

Thomas Wurmb, Katja Scholtes, Felix Kolibay, Peer Rechenbach, Ulrich Vogel, Barbara Kowalzik

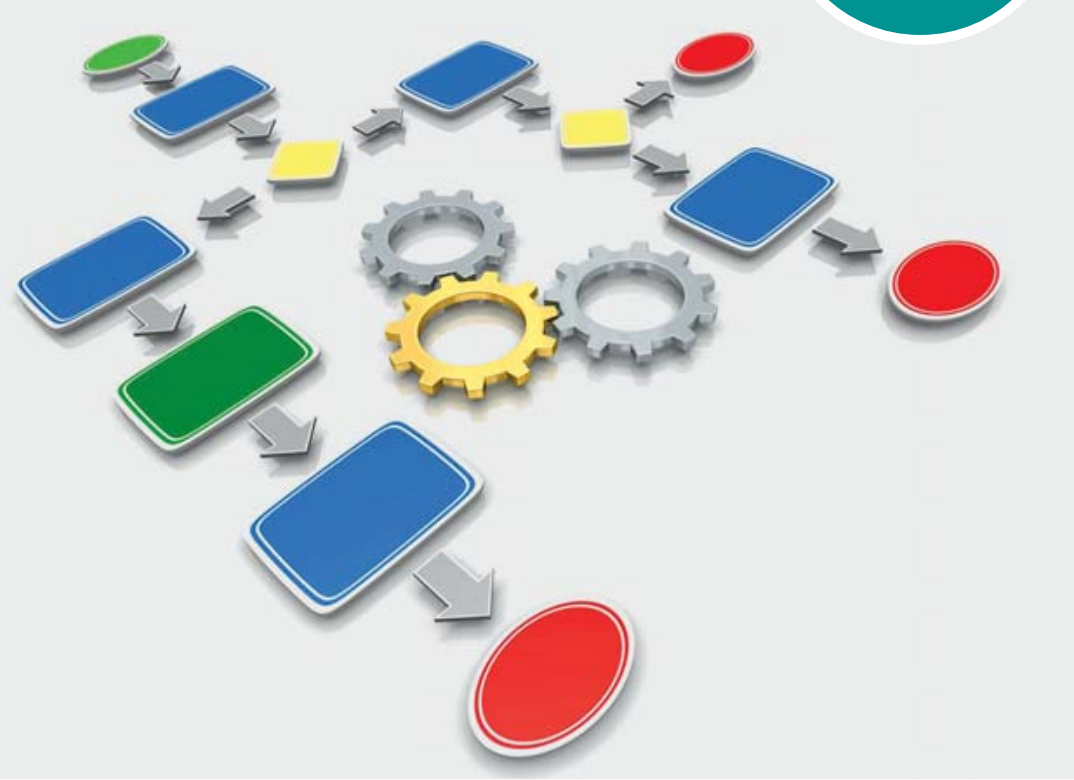

Krankenhäuser spielen bei der Bewältigung von Großschadenslagen oder Katastrophen eine entscheidende Rolle. Um dieser gerecht zu werden, bedarf es einer detaillierten Vorbereitung - der Erstellung eines Krankenhaus Alarm- und Einsatzplanes. Dieser Beitrag beschreibt die theoretischen Hintergründe der Alarm- und Einsatzplanung an Krankenhäusern. Darüber hinaus wird die konkrete Erstellung eines Einsatzplanes anhand eines Beispiels veranschaulicht.

\section{Hintergrund}

\section{Kritische Infrastrukturen}

Die Terroranschläge von Paris im November 2015 [1,2] haben die entscheidende Rolle der Krankenhäuser bei der Bewältigung einer hochkomplexen Situation verdeutlicht. Aber nicht nur solche Extremsituationen stellen Krankenhäuser vor große Herausforderungen. Auch ein Ausfall z. B. der elektrischen Energieversorgung oder der Wasserversorgung hat erhebliche Auswirkungen auf das Funktionieren der Abläufe im Krankenhaus. Darüber hinaus hat in der jüngsten Vergangenheit der Klinikbrand in Bochum gezeigt, dass Krankenhäuser selbst zum Ort des Schadensereignisses werden können. Die Auswirkungen eines solchen Ereignisses sind erheblich und zeigen die Verwundbarkeit von komplexen Institutionen, wie es Krankenhäuser sind. Krankenhäuser zählen zu den 9 Sektoren der Kritischen Infrastruktur, wie sie das Bundesamt für Bevölkerungsschutz und Katastrophenhilfe beschreibt [3]. Kritische Infrastrukturen (s. „Info - 9 Sektoren“) haben eine wichtige Bedeutung für das staatliche Gemeinwesen. Bei deren Ausfall oder Beeinträchtigung ist mit Versorgungsengpässen, erheblichen Störungen der öffentlichen Sicherheit oder anderen dramatischen Folgen zu rechnen [3]. 


\section{INFO}

\section{Sektoren}

Die Kritischen Infrastrukturen werden in folgende

Sektoren eingeteilt:

- Gesundheit

- Informationstechnik und Telekommunikation

- Wasser

- Transport und Verkehr

- Finanz- und Versicherungswesen

- Energie

- Ernährung

- Staat und Verwaltung

- Medien und Kultur

Krankenhäuser sind ein unverzichtbares Element der Daseinsvorsorge und haben eine herausragende Bedeutung im Bevölkerungsschutz. Besonders kritisch ist die Tatsache, dass Krankenhäuser aufgrund ihrer komplexen Anforderungen und zahlreichen Abhängigkeiten zu den verwundbarsten Infrastrukturen unseres Landes gehören [3]. Ein Ausfall oder eine nachhaltige Störung der stationären Krankenversorgung könnte jedes größere Schadensereignis zu einer schwer zu bewältigenden Katastrophe werden lassen [3].

Merke

Krankenhäuser zählen zu den Kritischen Infrastrukturen in Deutschland.

\section{Sicherstellung der Patientenversorgung}

Im Mittelpunkt der Aufgaben eines Krankenhauses steht die medizinische Patientenversorgung durch Ärzte und Pflegekräfte. Um einen störungsfreien Ablauf dieser Aufgabe zu gewährleisten, ist eine fein abgestimmte Koordination von Einzelprozessen mit einer höchst aufwendigen Technik und Logistik erforderlich. Eine einfache Störung der geregelten Routineabläufe in einem Krankenhaus hat das Potenzial, eine hochkomplexe Lage zu verursachen, die die Patientenversorgung und damit die Kernaufgabe gefährdet. Solche Störungen werden bestenfalls im Sinne der Prävention verhindert oder aber schnellstmöglich beseitigt, um den ursprünglichen Zustand wiederherzustellen.

Zum Schutz des Krankenhauses und zur Sicherstellung der Patientenversorgung ist eine umfassende Vorbereitung und Planung - die Krankenhaus Alarm- und Einsatzplanung (KAEP) - essenziell. Die gesetzlichen Regelungen hierzu sind bundesweit nicht einheitlich und finden sich in unterschiedlichen Gesetzen (z. B. Katastrophenschutzgesetze der Länder, Gesetz zur Kontrolle und Transparenz im Unternehmensbereich/KonTraG). Eine Übersicht bietet $>$ Tab. 1. Nach dem KonTraG gehört es zu den Pflichten eines Unternehmens, sich in geeigneter Weise auf potenzielle Ereignisse vorzubereiten, die entsprechende Risiken in sich bergen. Dies gilt selbstverständlich für Krankenhäuser gleichermaßen. Letztlich muss es im Interesse einer Krankenhausleitung liegen,

- Tab. 1 Übersicht über die gesetzlichen Regelungen zur Krankenhaus Alarm- und Einsatzplanung. Quelle: Willi Marzi.

\begin{tabular}{|l|l|}
\hline Bundesland & gesetzliche Grundlage \\
\hline Baden-Württemberg & Landeskrankenhausgesetz und Gesetz über den Katastrophenschutz \\
\hline Bayern & Bayerisches Katastrophenschutzgesetz \\
\hline Berlin & $\begin{array}{l}\text { Landeskrankenhausgesetz und Katastrophenschutzgesetz } \\
\text { Gesetz über den Brandschutz, die Hilfeleistung und den Katastrophenschutz des Landes Brandenburg (Brandenburgisches } \\
\text { Brand- und Katastrophenschutzgesetz) }\end{array}$ \\
\hline Brandenburg & Bremisches Krankenhausgesetz \\
\hline Bremen & Hamburgisches Krankenhausgesetz \\
\hline Hamburg & Hessisches Brand- und Katastrophenschutzgesetz und Hessisches Rettungsdienstgesetz \\
\hline Hessen & $\begin{array}{l}\text { Krankenhausgesetz für das Land Mecklenburg-Vorpommern und Gesetz über den Katastrophenschutz in Mecklenburg- } \\
\text { Vorpommern }\end{array}$ \\
\hline Mecklenburg- & Niedersächsisches Krankenhausgesetz \\
\hline Vorpommern & Krankenhausgestaltungsgesetz des Landes Nordrhein-Westfalen \\
\hline Niedersachsen & $\begin{array}{l}\text { Landesgesetz über den Brandschutz, die allgemeine Hilfe und den Katastrophenschutz (Brand- und Katastrophenschutz- } \\
\text { gesetz) und Landeskrankenhausgesetz }\end{array}$ \\
\hline Nordrhein-Westfalen & Saarländisches Krankenhausgesetz \\
\hline Rheinland-Pfalz & Sächsisches Krankenhausgesetz und Sächsisches Gesetz über den Brandschutz, Rettungsdienst und Katastrophenschutz \\
\hline Saarland & Krankenhausgesetz des Landes Sachsen-Anhalt und Katastrophenschutzgesetz des Landes Sachsen-Anhalt \\
\hline Sachsen & Gesetz über den Katastrophenschutz in Schleswig-Holstein \\
\hline Sachsen-Anhalt & Thüringer Krankenhausgesetz und Thüringer Gesetz über den Brandschutz, die Allgemeine Hilfe und den Katastrophenschutz \\
\hline Schleswig-Holstein & \\
\hline Thüringen &
\end{tabular}


- das eigene Unternehmen vor Schaden zu bewahren,

- eine optimale Patientenversorgung zu gewährleisten und

- bestmöglich auf Ausnahmesituationen vorzubereitet zu sein [4].

Merke

Krankenhäuser sind gesetzlich zu einer umfassenden Alarm- und Einsatzplanung verpflichtet.

\section{Verschiedene Modelle der KAEP}

\section{Traditionelle Einteilung: extern/intern}

Bei der traditionellen Gliederung von Krankenhaus Alarm- und Einsatzplänen werden Schadensereignisse in interne und externe Schadenslagen eingeteilt [4-6]. Die Einteilung basiert auf der Frage, wo das ursprüngliche Schadensereignis stattfindet. Hierbei wird der Massenanfall von Patienten üblicherweise zu den externen Ereignissen gezählt. Dagegen werden Ereignisse, die sich primär im Krankenhaus abspielen, z.B. ein Brandereignis oder Versorgungsausfälle (Ausfall der elektrischen Energieversorgung oder Informationstechnologie), zu den internen Schadenslagen gerechnet.

Diese Struktur bietet zunächst einmal eine gute Unterteilung, weist aber auch einige Nachteile auf. So gehört der Massenanfall von Patienten klassischerweise zu den externen Lagen, ein Brandereignis im Krankenhaus wird hingegen zu den internen Lagen gezählt. Bei einem Krankenhausbrand, der eine Vielzahl an Verletzten fordert, ist die ursprüngliche Zuordnung von „intern“ und „extern“ ungültig geworden. Letztlich ist diese Einteilung für die konkrete Bewältigung des Schadensereignisses ohne Relevanz (s. „Hintergrund - Vorbereitungszeit“).

\section{Hintergrund}

\section{Vorbereitungszeit}

Aus der rein beschreibenden Einteilung „intern/extern“ ergeben sich keine konkreten Konsequenzen für die Erstellung des Alarm- und Einsatzplanes. Selbst die Vorbereitungszeit des Krankenhauses muss bei externen Ereignissen nicht unbedingt länger sein als bei internen Ereignissen. So wurde ein Krankenhaus in Paris in der Anschlagsnacht vom 13.11.2015 erst durch schwer verletzte Selbsteinweiser auf das Anschlagsgeschehen aufmerksam - de facto war somit keinerlei zeitlicher Vorlauf gegeben [1].

Weitere Probleme ergeben sich beim Thema Einsatzführung. Die Gesamteinsatzleitung bei einer internen Lage liegt nicht grundsätzlich, wie man annehmen könnte, beim Krankenhaus selbst. Dies ist nur bei einigen internen Lagen der Fall, wie z. B. beim Ausfall der Strom- oder
Wasserversorgung. Bei einem Brandereignis am Krankenhaus liegt die Gesamteinsatzleitung bei der Feuerwehr, bei einem Ereignis mit kriminellem Hintergrund liegt die Einsatzleitung hingegen bei der Polizei. Die Trennung in interne und externe Ereignisse hat also auch in Bezug auf das Thema Einsatzführung keine Relevanz.

\section{Merke}

Die Einteilung des Krankenhaus Alarm- und Einsatzplanes in interne und externe Lagen bereitet Probleme - denn in Bezug auf die Klassifizierung wie auch in Hinblick auf die Einsatzführung müssen verschiedene Aspekte berücksichtigt werden.

\section{Konsequenzbasiertes Modell}

Das sog. konsequenzbasierte Modell wurde von Wurmb et al. kürzlich publiziert und verfolgt einen neuen Ansatz [7]. Nicht der Ort, an dem sich das Schadensereignis abspielt, steht hier im Mittelpunkt. Das Modell bezieht sich vielmehr auf die Konsequenzen, die sich durch das Ereignis für das Krankenhaus ergeben. Diese Konsequenzen kann man letztlich auf 2 wesentliche Merkmale reduzieren:

- die Reduktion/Überlastung der Versorgungskapazität oder

- die Störung der Funktionalität des Krankenhauses.

Zwischen beiden Merkmalen besteht ein direkter Zusammenhang. Eine Störung der Funktionalität hat im weiteren Verlauf auch eine Beeinträchtigung der Kapazität zur Folge. Umgekehrt bewirkt eine akute oder auch chronische Überlastung der Kapazität eine Einschränkung der Funktionalität [7]. Das Entscheidende bei dem konsequenzbasierten Ansatz ist, dass dieses Modell planerische Konsequenzen hat. Hierbei sind vor allem die Auseinandersetzung mit den konkreten Auswirkungen von Schadensereignissen und der Entwurf entsprechender Maßnahmenbündel zur Bewältigung dieser Auswirkungen von Bedeutung [7].

\section{Merke}

Das konsequenzbasierte Modell berücksichtigt die Störung der Funktionalität und die Reduktion der Behandlungskapazität als die beiden wichtigsten Auswirkungen einer Schadenslage auf ein Krankenhaus.

\section{Überlastung der Versorgungskapazität}

Eine Überlastung der Versorgungskapazität entsteht, wenn die Patientenzahlen stark und unvorhergesehen ansteigen [7]. Hierbei ist es kaum möglich, absolute Patientenzahlen festzulegen. Letztlich hängt das Eintreten einer Überlastung von zahlreichen Faktoren ab. Zum einen ist natürlich die Größe und Struktur des jeweiligen Hauses bedeutend. Zum anderen spielen aber Faktoren wie die Tageszeit, der Wochentag und die aktuelle Auslastung des jeweiligen Krankenhauses eine wichtige Rolle. Entscheidend für die Ausarbeitung des Einsatzplanes ist es, 
im Vorfeld Maßnahmen festzuschreiben, die zunächst darauf ausgerichtet sind, die ankommenden Patienten medizinisch angemessen zu versorgen. Die nächsten Schritte haben das Ziel, das Versorgungsangebot an den Versorgungsbedarf anzugleichen. Gelingt dies nicht, kommt es zu einer Störung der Funktionalität des Krankenhauses. Davon können in der Folge auch Bereiche betroffen sein, die mit dem auslösenden Ereignis eigentlich nichts zu tun haben. Ziel bei der Erstellung des KrankenhausAlarm- und Einsatzplanes ist es, dies zu verhindern.

\section{Störung der Funktionalität}

Eine Störung der Funktionalität tritt ein, wenn bauliche, technische, logistische, organisatorische oder personelle Voraussetzungen für die Patientenversorgung nicht mehr gegeben sind: z. B. bei einem Ausfall der Wasserversorgung, einem IT-Ausfall oder auch bei extremen Naturereignissen mit Gebäudeschäden.

Eine Störung der Funktionalität geht mit einer potenziellen Patientengefährdung einher. Daher muss es bei der Erstellung des Krankenhaus Alarm- und Einsatzplanes eine zentrale Rolle spielen,

- Gegenmaßnahmen festzulegen und

- Ausweich- und Alternativverfahren zur Wiederherstellung der gestörten Funktionalität zu benennen [7].

Merke

Ziele der KAEP sind:

- Erhalt der Funktionalität

- Aufrechterhaltung und Anpassung der Behandlungskapazität

- Schutz von Patienten, Mitarbeitern und weiteren Personen

\section{Ablauf der KAEP}

Im Folgenden wird der grundsätzliche Ablauf der KAEP dargestellt: die grundlegenden Voraussetzungen, die Rolle des Leiters der KAEP und eine Risikoanalyse. Darüber hinaus wird anhand des Massenanfalls von infizierten Patienten ein konkretes Beispiel für die Erstellung eines Planes vorgestellt.

\section{Voraussetzungen}

Eine wesentliche Voraussetzung für eine erfolgreiche KAEP ist die Bereitschaft der Krankenhausleitung, eine solche Planung zu etablieren und entsprechend zu unterstützen. Die konsequente Umsetzung erfordert finanzielle und personelle Ressourcen, die vonseiten der Krankenhäuser bereitgestellt werden müssen. Eine Gegenfinanzierung gibt es nicht, obwohl dies dringend zu fordern ist. Im Sinne einer möglichst guten Durchdringung der KAEP beim Personal ist es ebenfalls effektiv, dass die Krankenhausleitung der Planung ein adäquates Gewicht verleiht. Dies kann z. B. derart erfolgen, dass der gesamte Alarmund Einsatzplan als Dienstanweisung konzipiert ist.
Eine weitere wichtige Voraussetzung ist die Gestaltung einer Organisationsstruktur. Hierzu zählt, einen Leiter der KAEP zu benennen und eine (ständige) interdisziplinäre und multifunktionelle Arbeitsgruppe zu gründen. Die Komplexität eines Krankenhausbetriebes muss sich in der personellen Zusammensetzung einer solchen Arbeitsgruppe widerspiegeln. Vertreter der Ärzte, der Pflege, der Technik, der Logistik sowie des Brand- und Arbeitsschutzes sollen in dieser Arbeitsgruppe aktiv eingebunden sein - nur so ist eine fach- und sachkundige Erstellung des Planes zu gewährleisten. Die Verantwortung und gesamte Planung an einen einzelnen „Beauftragten“ zu übertragen, ist nach Ansicht der Autoren nicht mehr zeitgemäß.

Merke
Voraussetzung für eine funktionierende KAEP ist die
Unterstützung durch die Krankenhausleitung.

\section{Leiter der KAEP}

Die Erstellung der KAEP und die Führung der o.g. Arbeitsgruppe ist ein aufwendiger Prozess, für den es eine verantwortliche Person geben muss. Dieser Leiter muss von der Krankenhausleitung benannt und mit entsprechenden Kompetenzen (Weisungsbefugnis) ausgestattet werden. Die Bewältigung der vielfältigen Aufgaben erfordert zumindest eine partielle Freistellung von seinen alltäglichen klinischen Pflichten.

Für die erforderlichen Qualifikationen gibt es bisher keine einheitlichen Vorgaben. Die Deutsche Arbeitsgemeinschaft Krankenhaus Einsatzplanung (DAKEP e. V.) erarbeitet derzeit ein Curriculum, das sich noch im Entwurfsstadium befindet. Ungeachtet dessen lässt sich ein Profil herausarbeiten. Der Leiter KAEP sollte eine akademische Berufsausbildung mit Kenntnis der Kernprozesse eines Krankenhauses haben und er sollte über eine Führungsausbildung im Bereich Medizin verfügen. Für Ärzte wäre hier die Ausbildung zum Leitenden Notarzt nach den Empfehlungen der Bundesärztekammer zu nennen. Darüber hinaus sehr hilfreich - um die anspruchsvolle Aufgabe zu bewältigen - sind Kenntnisse

- aus der Notfall- und Katastrophenmedizin,

- über die Zusammenhänge zwischen Medizin, Technik und Logistik,

- aus Projekt-, Qualitäts- und Risikomanagement sowie

- allgemeine Kenntnisse des Arbeits- und Brandschutzes.

Merke

Die Leitung der KAEP braucht ein Team für eine erfolgreiche Bewältigung der zahlreichen Aufgaben.

\section{Erstellung des Krankenhaus Alarm- und Einsatzplanes}

Die Erstellung des Alarm- und Einsatzplanes verläuft in mehreren Phasen. An erster Stelle steht die Risikoanalyse. 


\section{Risikoanalyse}

Dieser Prozess ist in einer Broschüre des Bundesamtes für Bevölkerungsschutz und Katastrophenhilfe „Schutz kritischer Infrastruktur: Risikomanagement im Krankenhaus“ ausführlich beschrieben [3]. Die Risikoanalyse wird in 3 Schritte untergliedert:

1. Kritikalitätsanalyse: Zuerst werden kritische Prozesse in einem Krankenhaus, die für die Aufrechterhaltung der Behandlungskapazität und der Funktionalität von entscheidender Bedeutung sind, identifiziert.

2. Gefährdungsanalyse: Danach werden Gefahren analysiert und bewertet, die prinzipiell für das eigene Krankenhaus bestehen. Hierbei ist vor allem auch die Eintrittswahrscheinlichkeit in Betracht zu ziehen. So ist für ein Krankenhaus in einer Küstenlage die Wahrscheinlichkeit für eine Störung der Funktionalität durch Sturmschäden höher als für die Schädigung durch ein Schneelawinenunglück.

3. Verwundbarkeitsanalyse: Hierbei werden die Ergebnisse der Kritikalitätsanalyse und der Gefährdungsanalyse aufeinander bezogen und ausgewertet. Es entsteht so eine Matrix aus kritischen Prozessen auf der einen Seite und der Gefährdung dieser Prozesse durch spezifische Gefahren auf der anderen Seite.

Die höchste Priorität bei der KAEP haben Prozesse, die

- für die Funktionalität und Kapazität eines Krankenhauses besonders wichtig sind,

- gleichzeitig aber auch durch Gefahren mit einer hohen Eintrittswahrscheinlichkeit bedroht werden.

\section{Merke}

Vor der Erstellung des Krankenhaus Alarm- und Einsatzplanes muss eine Risikoanalyse durchgeführt werden.

\section{Projektplan}

Nach Abschluss der Risikoanalyse und einer entsprechenden Priorisierung muss als nächster Schritt durch den Leiter KAEP ein konkreter Projektplan entworfen werden. In welcher Form der Krankenhaus Alarm- und Einsatzplan erstellt wird, kann nicht einheitlich vorgegeben werden. Hier spielen die besonderen Gegebenheiten der einzelnen Krankenhäuser sowie länderspezifische Regelungen eine Rolle.

Bedeutsam sind eine übersichtliche Gliederung und eine gute Lesbarkeit. Darüber hinaus müssen hohe Verfügbarkeit und Vollständigkeit gewährleistet werden. Eine mögliche Struktur ist die Gliederung des gesamten Krankenhaus Alarm- und Einsatzplanes in ereignisspezifische Pläne (z. B. Massenanfall von Verletzten, polizeiliche Gefahrenlagen) und dazugehörige Handlungsanweisungen oder Checklisten. Diese Form ermöglicht

- in den ereignisspezifischen Plänen eine ausführliche Darstellung der Verantwortlichkeiten, der Raumord- nung, der Personalstruktur und der Hintergründe sowie

- mittels der Handlungsanweisungen eine wertvolle Unterstützung der handelnden Personen bei der Bewältigung des konkreten Einsatzes.

\section{Merke}

Bei der Erstellung des Krankenhaus Alarm- und Einsatzplanes sind eine übersichtliche Gliederung und die Erstellung von Checklisten und Auftragsblättern unverzichtbar.

Im Projektplan sind alle zu erstellenden Pläne enthalten (Beispiel s. „Praxis - Projektplan im Rahmen der KAEP“).

\section{PRAXIS}

\section{Projektplan im Rahmen der KAEP}

- Massenanfall von Patienten

- chirurgisch (Massenanfall von Verletzten, MANV)

- biologische Gefahrenlage, „B-Lage“ (infektiöse Patienten)

- chemische Gefahrenlage, „C-Lage“ (Intoxikation, Kontamination)

- radionukleare Gefahrenlage, „RN-Lage“

- Spezialfälle

- Patienten mit hochkontagiösen Erkrankungen

- Brandschutzpläne für sämtliche Gebäude

- Störung der Versorgung des Krankenhauses

- Ausfall der Informationstechnologie

- Ausfall der elektrischen Energieversorgung

- Ausfall der Wasserversorgung

- Ausfall der Wärmeversorgung

- Ausfall der Telekommunikation

- polizeiliche Lagen

- Amoklauf

- Bombendrohung

- Erpressung

- Geiselnahme

- vermisster Patient

- Entführung

\section{Ereignisspezifische Pläne}

Mit der Ausarbeitung der ereignisspezifischen Pläne wird begonnen, sobald der gesamte Projektplan fertiggestellt und eine prioritätenorientierte Zeitschiene erstellt ist. Hierzu muss der Leiter KAEP entsprechende Expertengruppen zusammenstellen. Diese sollen so zusammengesetzt sein, dass Vertreter aller betroffenen Kliniken und Abteilungen an der Entstehung des Planes beteiligt werden. Nur so kann gewährleistet werden, dass alle wesentlichen Aspekte zur Bewältigung des Ereignisses berücksichtigt werden. 


\section{Erstellung eines ereignisspezifischen Planes am Beispiel einer B-Lage}

Die konkrete Umsetzung wird nun am Beispiel eines ereignisspezifischen Planes für den Massenanfall von infektiösen Patienten (B-Lage) an einem Krankenhaus der Maximalversorgung dargestellt.

\section{PRAXIS}

\section{Expertengruppe B-Lage}

An der Erstellung eines ereignisspezifischen Planes für eine B-Lage sollten Führungskräfte folgender Disziplinen beteiligt sein:

- Innere Medizin

- Notaufnahme

- Infektiologie

- Krankenhaushygiene

- Mikrobiologie

- Anästhesie

- Chirurgie

- Intensivmedizin

- Logistik

- Technik

- Apotheke

- Labor, Zentralsterilisation

- Lager

- Reinigungsdienst

- Verwaltung und Personalrat

Die Expertengruppe für eine B-Lage sollte sich aus entscheidungsbefugten (Führungskräfte) Vertretern verschiedener Fachrichtungen zusammensetzen (s. Praxis).

Als externe Berater oder Teilnehmer müssen Vertreter des Öffentlichen Gesundheitsdienstes, des Rettungsdienstes und ggf. der Feuerwehr mit einbezogen werden.

\section{Grundprinzipien}

Bei der Erstellung des Planes müssen zunächst einige Grundprinzipien beachtet und festgelegt werden. Der Massenanfall von infizierten Patienten bezeichnet eine Situation, in der eine große Zahl von infizierten und infektiösen Patienten versorgt werden muss. Ziel dieses Einsatzplanes ist es, einen geordneten Einsatzablauf am Krankenhaus im Falle einer B-Lage zu gewährleisten.

Merke

Eigenschutz, der Schutz des Krankenhauses und der dort befindlichen Patienten haben einen hohen Stellenwert bei einer B-Lage.
Bei einem Massenanfall von infizierten Patienten handelt es sich in erster Linie um eine Überlastung der Behandlungskapazität. Alle planerischen Bemühungen müssen darauf ausgerichtet sein, die Behandlungskapazität möglichst schnell wieder an den Bedarf anzupassen. Dies muss stattfinden, bevor es über das Schadensereignis hinaus zu einer nachhaltigen Störung der Funktionalität kommt.

Ziel des Planes zur Bewältigung einer B-Lage ist es, eine abgesonderte Sichtung, Isolierung und Behandlung der ankommenden Patienten und ihrer Kontaktpersonen sicherzustellen. Eine Verbreitung von Erregern in größeren Teilen des Krankenhauses ist unbedingt zu vermeiden. Der Schutz des Personals und der vorhandenen Patienten hat eine sehr hohe Priorität. Gegebenenfalls muss eine Dekontamination der Patienten durchgeführt werden, die es zu planen gilt (s. „Hintergrund - Dekontamination von Patienten“).

\section{HINTERGRUND \\ Dekontamination von Patienten \\ Die wichtigste Dekontaminationsmaßnahme ist das Entfernen der Kleidung. Die weitere Dekontamina- tion erfolgt durch das Abspülen mit Wasser oder durch Abduschen. In Deutschland verfügen aller- dings nur wenige Krankenhäuser über die Möglich- keit zur Dekontamination einer Vielzahl von Patien- ten. Das Vorhalten solcher Anlagen erfordert einen hohen Aufwand, nicht zuletzt auch an Schulungen. Absprachen mit der Feuerwehr oder auch Einheiten des Katastrophenschutzes sind notwendig, um das Vorgehen in einer solchen Lage im Vorfeld zu regeln. Denn sowohl die Feuerwehr als auch der Katastro- phenschutz verfügen über Möglichkeiten zur Dekon- tamination.}

Eine frühe Isolierung der eintreffenden Patienten ist unbedingt erforderlich. Die Patientenströme werden so geregelt, dass es zu möglichst wenigen Kreuzungspunkten (dazu zählen auch Geräte, Instrumente und diagnostische Einrichtungen) mit nicht betroffenen Patienten kommt. Hausinterne Verlegungen von betroffenen Patienten sollten vor allem in der Initialphase auf das notwendige Minimum reduziert sein.

Bei Verdacht auf das Vorliegen einer hochkontagiösen Erkrankung (z. B. Ebola) gilt ein gesonderter Alarmplan für hochkontagiöse Infektionskrankheiten. Hierzu gibt es die Vorgaben des STAKOB (Ständiger Arbeitskreis der Kompetenz- und Behandlungszentren für hochkontagiöse und lebensbedrohliche Erkrankungen beim Robert Koch-Institut). 


\section{Strukturelemente}

Die folgenden Strukturelemente müssen in dem zu erstellenden Plan enthalten sein.

\section{Alarmierung}

Es muss festgelegt werden, wie eine Alarmierung im Falle einer B-Lage vonstattengeht. Es ist sinnvoll, hierbei auf alltäglich etablierte Meldewege zurückzugreifen. Die Alarmierung muss an einer Stelle im Krankenhaus eingehen, die rund um die Uhr besetzt ist.

Merke

Es muss gewährleistet sein, dass ein Entscheidungsträger die Alarmierung entgegennimmt, damit unmittelbar Konsequenzen und eine Aktivierung des Alarmplanes „B-Lage“ erfolgen können.

\section{Bildung einer Klinikeinsatzleitung}

Zur Bewältigung von komplexen Einsatzlagen ist es essenziell, frühestmöglich eine Führungsstruktur aufzubauen. Dies gilt auch für die Bewältigung einer B-Lage. Im Plan muss festgelegt werden, aus welchen Funktionen sich die Klinikeinsatzleitung (KEL) zusammensetzt und wie diese Funktionen alarmiert werden. Es empfiehlt sich, die Mitglieder der KEL aus den Bereichen Ärztlicher Dienst, Pflegedienst, Technik und Logistik zu rekrutieren. Es muss ein Einsatzort für die KEL definiert werden, dort sollten auch entsprechende Kommunikationsmittel (z. B. reservierte Mobiltelefone) zur Verfügung gestellt werden. Eine Kennzeichnung der Funktionen innerhalb der KEL empfiehlt sich. Ab einer gewissen Größe des Schadensereignisses muss am Krankenhaus eine stabsähnliche Führungsstruktur etabliert werden. Wie dies vonstattengeht, welche Funktionen hier besetzt werden und wer die Gesamteinsatzleitung übernimmt, muss gesondert für das jeweilige Krankenhaus festgelegt werden.

\section{Raumordnung}

Gerade bei der Bewältigung einer B-Lage ist eine klar definierte Raumordnung mit der Möglichkeit zur Isolierung und zur Dekontamination von entscheidender Bedeutung. Nur so können die Ziele „Schutz des Krankenhauses“, „Schutz der nicht betroffenen Patienten“ und „Schutz des Personals“ erreicht werden. Die Anfahrtswege des Rettungsdienstes, eine Sichtungsstelle, eine Dekontaminationsmöglichkeit und die Behandlungsräume müssen festgelegt werden. Wichtig ist hier die klare Trennung von „unreinen“ und „reinen“ Raumabschnitten. Je nach räumlichen Gegebenheiten muss über die Etablierung einer Ersatznotaufnahme nachgedacht und diese im Vorfeld definiert werden. Wichtig hinsichtlich der Raumordnung ist es auch, über eine ausreichende Materialbevorratung und die anschließende Materialentsorgung nachzudenken. Die Reinigung und Desinfektion der Räume muss im Vorfeld geplant werden.

\section{Personal}

Gerade in der Frühphase eines solchen Einsatzes wird es zu einem Ungleichgewicht zwischen eintreffenden Patienten und vorhandenem Personal kommen. Im Plan muss festgelegt werden, wie diese Überlastung der Kapazität schnellstmöglich behoben werden soll. Zunächst muss mit dem vorhandenen Personal eine Struktur aufgebaut werden, um die eintreffenden Patienten zu sichten und dann gemäß dem Sichtungsergebnis schnellstmöglich zu behandeln.

Die Kräfte können aufgestockt werden, indem Personal aus den nicht betroffenen Kliniken (einschließlich der Rufdienste außerhalb der regulären Arbeitszeit) rekrutiert wird. Dies muss im Vorfeld interdisziplinär besprochen und festgeschrieben werden. Ebenso empfiehlt es sich, für dieses „fachfremde“ Personal die zu übernehmenden Aufgaben im Vorfeld zu definieren. Um die Verfügbarkeit von Personal und damit die Behandlungskapazität weiter zu erhöhen, kann es nötig werden, den Routinebetrieb einzelner Kliniken oder des gesamten Krankenhauses vorübergehend zu reduzieren. Dies sind einschneidende Maßnahmen für ein Krankenhaus und seine Patienten und bedeutet eine deutliche Einschränkung der Funktionalität.

Darüber hinaus muss auf dienstfreies Personal zurückgegriffen werden. Der Modus der Alarmierung, der technische Vorgang und die Mitarbeiterdatenbank mit den Erreichbarkeiten müssen im Vorfeld detailliert festgelegt und kommuniziert werden. Eine elektronische Alarmierung über einen automatischen Alarmierungsserver empfiehlt sich, muss aber entsprechend etabliert und gepflegt werden. Im Zuge der Alarmierung von dienstfreiem Personal müssen Anfahrtswege und Sammelpunkte für die einrückenden Mitarbeiter definiert werden. Um einen geordneten Einsatz des Personals zu gewährleisten, muss ein Abschnittsleiter für die Registrierung und Einweisung des Personals eingesetzt werden.

\section{Merke}

Im Plan müssen Maßnahmen festgelegt werden, die das Ungleichgewicht zwischen vorhandenem und benötigtem Personal schnellstmöglich beheben können.

\section{Material}

Für die Bewältigung einer B-Lage sind die Bevorratung von Material und ein Konzept zur Entsorgung essenziell. Wichtig sind ausreichende Mengen von

- persönlicher Schutzausrüstung (PSA) entsprechend den erregerspezifischen Anforderungen,

- Desinfektionsmittel (Haut, Fläche, Instrumente) sowie

- Müll- und Wäschesäcken.

Es muss ein Konzept zur Bevorratung von Medikamenten (z. B. Antibiotika, Antiemetika) und Infusionslösungen er- 
stellt werden. Ebenso muss im Vorfeld abgesprochen werden, welche Diagnostik zum Einsatz kommt, wie die Proben gewonnen und verpackt werden und wie der Transport organisiert wird.

\section{Kommunikation}

Für die Bewältigung einer Großschadenslage ist eine funktionierende Kommunikation von großer Bedeutung. Hierzu zählt die Kommunikation innerhalb des Krankenhauses, aber auch die Kommunikation nach extern.

Für die interne Kommunikation müssen die Mittel und Wege im Vorfeld festgelegt werden. Dies kann z. B. über reservierte Telefone für die Mitglieder der KEL und Erreichbarkeitslisten erfolgen. Bei der internen Kommunikation darf auch die Information der stationären Patienten, des Krankenhauspersonals und deren Angehörigen nicht übersehen werden.

Zur Kommunikation nach extern zählen die Verbindung zur Rettungsleitstelle, ggf. auch zur Polizei, zum Öffentlichen Gesundheitsdienst und die Pressearbeit. Zur Bewältigung der Information von Angehörigen der vom Schadensereignis betroffenen Patienten könnte die Einrichtung und Besetzung von „Infotelefonen für Angehörige“ beitragen. Nicht zu vergessen ist die Abstimmung mit den anderen Krankenhäusern vor Ort. Ein gemeinsames Konzept und eine Kommunikation während eines Schadensereignisses können dazu beitragen, die Überlastung der Kapazität (z. B. durch Weiterverlegung von nicht betroffenen Patienten) zu reduzieren.

\section{Verfügbarkeit, Verstetigung und Übung}

\section{Verfügbarkeit}

Ein hoher Durchdringungsgrad des Krankenhaus-Alarmund Einsatzplanes bei den Mitarbeitern ist von größter Bedeutung für eine erfolgreiche Einsatzbewältigung. Eine Voraussetzung für eine gute Durchdringung ist die Verfügbarkeit des Alarm- und Einsatzplanes. Sowohl eine Papierversion als auch eine elektronische Form sollten vorhanden sein. Bei den Exemplaren in Papierform ist darauf zu achten, dass diese an den jeweiligen Einsatzschwerpunkten verfügbar sind. Neben der Schwierigkeit, diese Druckexemplare aktuell zu halten (sorgfältige Dokumentenlenkung), ist die Gefahr des Missbrauchs zu bedenken. Diese Gefahr besteht natürlich auch bei der elektronischen Form - dennoch ist die elektronische Verfügbarkeit wichtig. Um einen gewissen Schutz der Dokumente vor Missbrauch zu erreichen, kann die elektronische Form mit Nutzungsrechten belegt werden.

Merke

Der Krankenhaus Alarm- und Einsatzplan sollte sowohl elektronisch als auch in Papierform verfügbar sein.

\section{Übungen}

Übungen sind ein wichtiges Werkzeug. Sie dienen dazu, die erstellten Pläne auf ihre Tauglichkeit zu überprüfen, die einzelnen Schritte und Abläufe zu trainieren und die Schulung der Mitarbeiter zu gewährleisten. Übungen sind mit einem erheblichen Aufwand verbunden. Es bedarf einer detaillierten Planung und Vorbereitung, die tatsächliche Ausführung muss zusätzlich zum Routinebetrieb erfolgen und ist mit entsprechenden Kosten verbunden. Um einen Lerneffekt zu erzielen, der den hohen Aufwand auch rechtfertigt, bedarf es klar definierter Ziele, kundiger Beobachter und einer strukturierten Auswertung. Dabei ist es nicht unbedingt erforderlich, in jedem Fall eine Vollübung durchzuführen - auch die Übung einzelner Teilkomponenten kann effektiv sein und zur besseren Durchdringung der Pläne beitragen.

Merke
Übungen sind ein wichtiger Erfolgsfaktor für die
KAEP, sind jedoch mit einem großen Aufwand für
die Krankenhäuser verbunden.

HINTERGRUND

Gesetzliche Regelungen

In einigen Ländern/Stadtstaaten wie Hessen, Berlin und Hamburg gibt es eine gesetzliche Verpflichtung für die Krankenhäuser zur Mitwirkung an oder Durchführung von Übungen. In Hessen z. B. werden die Übungen seitens des Gesundheitsministeriums organisiert, teilweise finanziert und oft ohne Ankündigung durchgeführt. Das Gesundheitsressort unterstützt die Krankenhäuser bei der Auswertung und erstellt vertrauliche Berichte für die Krankenhäuser. In Berlin übernimmt diese Aufgabe die Senatsverwaltung für Gesundheit, Pflege und Gleichstellung, in Hamburg die Behörde für Gesundheit und Verbraucherschutz. In vielen anderen Bundesländern gibt es keine derartige behördliche Unterstützung. Den Krankenhäusern bleibt es selbst überlassen, Übungen zu organisieren, durchzuführen und auszuwerten.

\section{Verbesserungsprozess}

Die Erkenntnisse aus den Übungen sind wichtig, um die Alarm- und Einsatzpläne einem kontinuierlichen Verbesserungsprozess zu unterziehen. Dieser Prozess ist erforderlich, um eine Aktualität der Pläne zu garantieren. Die Pläne fortzuschreiben und kontinuierlich an neue Risiken und Bedrohungen anzupassen, ist eine nicht zu unterschätzende Aufgabe, die ebenfalls der Leiter KAEP zu bewältigen hat. 
Merke

Die Erstellung des Krankenhaus Alarm- und Einsatzplanes ist ein kontinuierlicher Prozess, der stetig fortgesetzt werden muss.

\section{Zusammenarbeit mit Rettungsdienst, Katastrophenschutz, Feuerwehr}

\section{Anfahrtswege}

Bei der Bewältigung von Großschadenslagen spielt der Rettungsdienst eine entscheidende Rolle. Die Anfahrtswege des Rettungsdienstes zum aufnehmenden Krankenhaus und die Einrichtung einer Übergabestelle sind wichtige Faktoren, die zur erfolgreichen Bewältigung eines Massenanfalls von Patienten beitragen. Idealerweise erfolgt die Einlieferung der Patienten über eine vorher festgelegte Zufahrt. Die Einrichtung eines Kreisverkehrs oder die Lösung mit Ein- und Ausgang im Einbahnstraßensystem sollte im Vorfeld geplant und gemeinsam mit Vertretern des Rettungsdienstes festgelegt werden. Entscheidendes Ziel ist die schnelle und reibungslose Übergabe der Patienten zwischen Rettungsdienst und Krankenhaus. Eine Verstopfung der Zu- und Abfahrtswege muss unter allen Umständen vermieden werden.

Bei einem Massenanfall von Patienten - insbesondere im Rahmen einer lebensbedrohlichen Einsatzlage (z. B. Terroranschlag) - müssen die Fahrzeuge des Rettungsdienstes schnellstmöglich wieder einsatzbereit sein. Es wird ihnen daher nicht gelingen, ihre Heimatwache anzufahren, um sich mit neuem Verbrauchsmaterial auszustatten. Ein Konzept könnte darin bestehen, seitens der Krankenhäuser fertig gepackte Sets vorzuhalten, die vom Rettungsdienst aufgenommen werden, um so eine schnelle Einsatzbereitschaft herzustellen. Dieses Vorgehen muss im Vorfeld geplant, abgestimmt und hinsichtlich der Finanzierung geregelt werden.

\section{Merke}

Die Planungen hinsichtlich der Zu- und Abfahrtswege sowie Patientenaufnahme müssen unbedingt mit dem Rettungsdienst abgestimmt werden, damit im Einsatzfall die Wege und Vorgehensweisen bekannt sind.

Für den Fall einer Gefährdung des Krankenhauses selbst müssen Rahmenbedingungen für den rettungsdienstlichen Einsatz definiert werden. Hierzu zählen die Festlegung von Anfahrtswegen, von Bereitstellungsräumen, von Patientenübergabestellen und die Planung von möglichen Evakuierungen.

\section{Feuerwehr}

Im Rahmen der KAEP ist die Feuerwehr ein unverzichtbarer Partner. So sollte beispielsweise bei der Erstellung von Brandalarmplänen ein Vertreter der Feuerwehr als ständiges Mitglied in der Expertengruppe eingebunden werden.
Dadurch wird sichergestellt, dass neben den brandschutztechnischen Fragen auch die Konzepte zur Räumung, Evakuierung und die Planung der Ablauforganisation bei einem Brand fachkompetent erstellt werden. Durch den ständigen Austausch mit der Feuerwehr kann unterschiedliches Problembewusstsein im Vorfeld identifiziert, kommuniziert und ggf. aufeinander abgestimmt werden.

\section{KERNAUSSAGEN}

- Die Krankenhaus Alarm- und Einsatzplanung (KAEP) ist ein aufwendiger Prozess. Sie bedarf der Unterstützung durch die Krankenhausleitung.

- Ein Leiter KAEP muss benannt werden. Dieser benötigt Unterstützung, Zeit und Befugnisse für eine erfolgreiche Umsetzung der Alarm- und Einsatzplanung.

- Wegen der hohen Komplexität eines modernen Krankenhauses muss die KAEP Vertreter aus Medizin, Technik, Logistik, Versorgung und Verwaltung mit einbeziehen.

- Der Alarm- und Einsatzplan muss von spezifischen Expertengruppen unter der Gesamtführung des Leiters KAEP erstellt werden.

- Am Anfang der konkreten Planerstellung steht eine Risikoanalyse.

- Die KAEP ist ein kontinuierlicher Prozess, der stetig fortgesetzt werden muss.

- Ausbildung und Übungen sind ein wichtiger Erfolgsfaktor für eine funktionierende KAEP.

- Die Zusammenarbeit mit Rettungsdienst, Katastrophenschutz und Feuerwehr ist ein unverzichtbarer Bestandteil der KAEP.

Interessenkonflikt

Die Autoren geben an, dass kein Interessenkonflikt besteht.

Danksagung

Wir danken Herrn Dr. Willi Marzi für die Überlassung der $\$ Tab. 1.

Über die Autoren

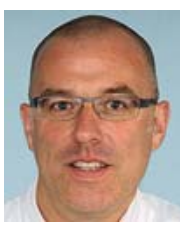

\section{Thomas Wurmb}

Univ.-Prof. Dr. med., Jahrgang 1969. Medizinstudium in Heidelberg. Seit 1997 an der Klinik und Poliklinik für Anästhesiologie Universitätsklinikum Würzburg. 2015 Berufung zum Universitätsprofessor für Notfall- und Katastrophenmedizin. Facharzt für Anästhesiologie, Zusatzbezeichnungen Notfallmedizin und Spezielle Anästhesiologische Intensivmedizin. Vorstandsmitglied der Deutschen Arbeitsgemeinschaft Krankenhaus Einsatzplanung (DAKEP e. V.), Leiter der AG Trauma- und Schockraummanagement des Arbeitskreises Notfallmedizin der DGAI. 


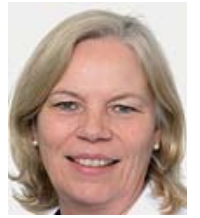

\section{Katja Scholtes}

Dr. med., Jahrgang 1956. Ausbildung zur Krankenschwester, Medizinstudium in Köln und Aachen, danach 20 Jahre als Ärztin im UK Aachen tätig. Seit 2016 Leitende Abteilungsärztin der Zentralen Notaufnahme des Krankenhauses Merheim der Kliniken der Stadt Köln. Fachärztin für Anästhesie und Notfallmedizin, Master of Health Business Administration und Master of Disaster Management and Risk Governance. Frau Dr. Scholtes ist Vorstandsvorsitzende der Deutschen Arbeitsgemeinschaft Krankenhaus Einsatzplanung (DAKEP e.V.)

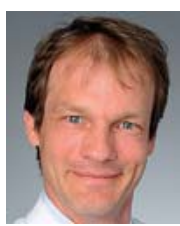

\section{Felix Kolibay}

Dr. med., Jahrgang 1968. Medizinstudium in Köln. Facharzt für Anästhesiologie und Zusatzbezeichnung Spezielle Anästhesiologische Intensivmedizin. Seit 1996 als Arzt an der Uniklinik Köln tätig. Verantwortlich für die Krankenhausalarm- und Einsatzplanung der Uniklinik Köln und Vorstandsmitglied der Deutschen Arbeitsgemeinschaft Krankenhaus Einsatzplanung (DAKEP e.V.).

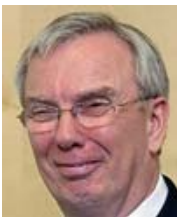

\section{Peer Rechenbach}

Prof. Dr.-Ing., Jahrgang 1952. Studium der Ingenieurwissenschaften im Fachbereich Elektrotechnik, 2004 Promotion, seit 2013 Professor der Hochschule für Angewandte Wissenschaften in Hamburg. Lehrbeauftragter an der Universität Bonn. Langjährige Tätigkeit bei der Feuerwehr Hamburg. 2007-2013 Leitung der Abteilung für Grundsatzangelegenheiten des Brandschutzes, des Rettungsdienstes, der Kampfmittelbeseitigung, Katastrophen-, Zivilund Bevölkerungsschutz sowie zivil-militärische Zusammenarbeit in der Behörde für Inneres und Sport in Hamburg.

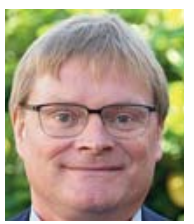

\section{Ulrich Vogel}

Univ.-Prof. Dr. med., Jahrgang 1964. Medizinstudium in Würzburg, Weiterbildung zum Facharzt für Mikrobiologie, Infektionsepidemiologie und Virologie in Hannover. Seit 1996 am Institut für Hygiene und Mikrobiologie der Universität Würzburg. Seit 2008 Krankenhaushygieniker, seit 2012 Leiter der neu gegründeten Stabsstelle Krankenhaushygiene. Leiter des Nationalen Referenzzentrums für Meningokokken und Haemophilus influenzae, das am Institut für Hygiene und Mikrobiologie der Uni Würzburg angesiedelt ist.

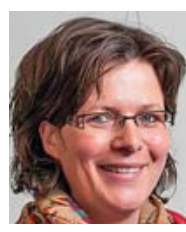

\section{Barbara Kowalzik}

Dr. med., MSc, Jahrgang 1971. 1990 Eintritt in den Sanitätsdienst der Bundeswehr. Medizinstudium in Hannover. Danach Sanitätsoffizier im Zentralen Sanitätsdienst der Bundeswehr, Notärztin im Kosovo und in Afghanistan. Seit 2008 Leitung des Referats Schutz der Gesundheit im Bundesamt für Bevölkerungsschutz und Katastrophenhilfe mit den Arbeitsschwerpunkten Sanitätsmaterialbevorratung, Krankenhausalarmplanung, MANV-Management und Seuchenhygienisches Management. Fachärztin für Allgemeinmedizin und Master of Health Management.
Univ.-Prof. Dr. med. Thomas Wurmb

Leiter der Sektion Notfall- und Katastrophenmedizin

Klinik und Poliklinik für Anästhesiologie

Oberdürrbacherstraße 6

97080 Würzburg

Wurmb_t@ukw.de

\section{Wissenschaftlich verantwortlich} gemäß Zertifizierungsbestimmungen

Wissenschaftlich verantwortlich gemäß Zertifizierungsbestimmungen für diesen Beitrag ist Univ.-Prof. Dr. med. Thomas Wurmb, Würzburg.

\section{Literatur}

[1] Haug C]. Report from Paris. N Engl] Med 2015; 373: 25892593

[2] Hirsch M, Carli P, Nizard R et al.; health professionals of Assistance Publique Hôpitaux de Paris (APHP). The medical response to multisite terrorist attacks in Paris. Lancet 2015; 386: 2535-2538

[3] Bundesamt für Bevölkerungsschutz und Katastrophenhilfe (BBK). Schutz kritischer Infrastruktur: Risikomanagement im Krankenhaus. 2008. Im Internet: http://www.bbk.bund.de/ DE/AufgabenundAusstattung/Kritischelnfrastrukturen/Publikationen/Leitfaden_Krankenh_Risiko-Kritis.html; Stand: 29.06.2017

[4] Adams HA, Flemming A, Krettek C et al. Der Notfallplan des Krankenhauses. Med Klin Intensiv Notfallmed 2015; 110: 37 48

[5] Cwoijdzinski D, Kammel P, Schneppenheim UW, Suckau M, UIbrich T, Hrsg. Leitfaden Krankenhaus Alarmplanung. Berlin: Fachverlag Matthias Grimm; 2008 (vergriffen)

[6] Adams HA, Flemming A, Hidebrand F et al. Der Notfallplan des Krankenhauses. Anästh Intensivmed 2012; 53: 62-81

[7] Wurmb T, Rechenbach P, Scholtes K. Alarm- und Einsatzplanung an Krankenhäusern: Das konsequenzbasierte Modell. Med Klin Intensivmed Notfallmed 2016; Jul 4. [Epub ahead of print]. doi:10.1007/s00063-016-0190-8

Bibliografie

DOI https://doi.org/10.1055/s-0042-120230

Anästhesiol Intensivmed Notfallmed Schmerzther 2017; 52 594-605 () Georg Thieme Verlag KG Stuttgart · New York ISSN 0939-2661 


\section{Punkte sammeln auf CME.thieme.de}

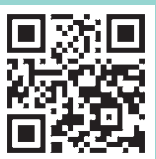

Diese Fortbildungseinheit ist 12 Monate online für die Teilnahme verfügbar.

Sollten Sie Fragen zur Online-Teilnahme haben, finden Sie unter cme.thieme.de/hilfe eine ausführliche Anleitung. Wir wünschen viel Erfolg beim Beantworten der Fragen!

Unter eref/thieme.de/ZZWHM6A oder über den QR-Code kommen Sie direkt zum Artikel zur Eingabe der Antworten.

VNR 2760512017152374023

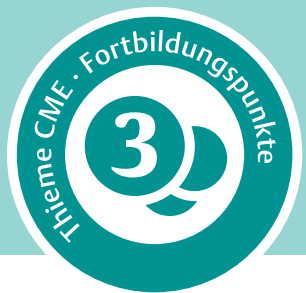

\section{Frage 1}

Welche Aussage ist richtig? Der Leiter der Krankenhaus-Alarmund Einsatzplanung (Leiter KAEP) ...

A muss als Stabsstelle an die Klinikleitung angebunden werden.

B muss von keinen seiner sonstigen Pflichten freigestellt werden.

C sollte auf jeden Fall ein Mediziner sein.

D sollte über Kenntnisse aus der Notfall- und Katastrophenmedizin verfügen.

E hat keine spezielle Weisungsbefugnis gegenüber Mitarbeitern des Krankenhauses.

\section{Frage 2}

Welche Aussage ist richtig?

A Die Erarbeitung der KAEP ist problemlos in den klinischen Alltag zu integrieren.

B Es existiert keine gesetzliche Verpflichtung, einen Krankenhaus-Alarm- und Einsatzplan zu erstellen.

C Besondere Lagen sind extrem selten, eine Planung ist unnötig.

D Die Durchführung von Übungen ist ein wichtiger Erfolgsfaktor für das Gelingen einer KAEP.

E Einmal erstellt, bedarf die KAEP keiner weiteren Bearbeitung.

\section{Frage 3}

Welche Aussage ist richtig?

A Die Unterteilung in interne und externe Schadenslagen ist sinnvoll und sollte immer die Grundlage bei der Erstellung eines Krankenhausalarmplanes bilden.

B Das sog. „konsequenzbasierte Modell“ berücksichtigt ausschließlich die Störung der Funktionalität.

C Bei internen Schadenslagen hat die Einsatzleitung immer das Krankenhaus.

D Die Überlastung der Versorgungskapazität ist unabhängig von Faktoren wie Tageszeit und Wochentag.

E Ereignisspezifische Pläne mit dazugehörigen Checklisten sind eine sinnvolle Struktur für die Gliederung eines Krankenhausalarmplanes.

\section{Frage 4}

Welche Aussage ist falsch?

A Die Formierung einer Klinikeinsatzleitung sollte im Schadensfall so schnell wie möglich erfolgen.

B Alarmierungswege sollten möglichst einfach gehalten sein.

C Bei komplexen Einsätzen ist das Hinzuziehen von Spezialisten wichtig.

D Die Alarm- und Einsatzpläne sollten gut verfügbar sein, unter Beachtung einer Missbrauchsgefahr.

E Spezielle Kommunikationsmittel für die Klinikeinsatzleitung sind nicht nötig.

\section{Frage 5}

Welche Aussage in Bezug auf den Massenanfall von infizierten Patienten ist falsch?

A Bei der Erstellung eines Planes für den Massenanfall von infizierten Patienten ist der Schutz von Personal und anderen Patienten ein wichtiges Ziel.

B Innerklinische Transporte von infektiösen Patienten sollten soweit wie möglich vermieden werden.

C Eine Sichtungsstelle für die eintreffenden Patienten muss eingerichtet werden.

D Die Einrichtung einer Ersatznotaufnahme ist nicht notwendig.

E Die Einbindung des Öffentlichen Gesundheitsdienstes sollte frühzeitig erfolgen.

\section{Frage 6}

Welche Aussage ist richtig?

A Die KAEP ist verbindlich in einem einzigen Gesetz geregelt.

B Die Verpflichtung zur Übung der Pläne ergibt sich aus einem Bundesgesetz.

C Übungen werden für die Krankenhäuser in allen Bundesländern seitens der Behörden organisiert und finanziert.

D Oftmals obliegt die Organisation und Durchführung von Übungen den Krankenhäusern.

E Eine Übung muss als Vollübung abgehalten werden, die Übung einzelner Teilaktionen ist nicht sinnvoll. 


\section{Punkte sammeln auf CME.thieme.de}

\section{Fortsetzung $\ldots$}

\section{Frage 7}

Welche Aussage ist falsch?

A Bei der Erstellung von Alarm- und Einsatzplänen ist eine Risikoanalyse an den Anfang zu stellen.

B Die Risikoanalyse umfasst eine Kritikalitätsanalyse.

C Die Risikoanalyse umfasst eine Gefährdungsanalyse.

D Die Risikoanalyse umfasst eine Häufigkeits- und Seltenheitsanalyse.

E Die Risikoanalyse umfasst eine Verwundbarkeitsanalyse.

\section{Frage 8}

Welche Aussage ist richtig?

A Ein Massenanfall von Verletzten (MANV) wird dem Krankenhaus immer durch die Rettungsleitstelle angekündigt.

B Für die Bewältigung eines MANV werden nur Ärzte und Pflegekräfte benötigt.

C Die Krankenhauslogistik und -technik sind wichtige Partner bei der Erstellung von Krankenhaus-Alarm- und Einsatzplänen.

D Der Leiter KAEP durchläuft eine klar geregelte Ausbildung.

E Die erforderliche Qualifikation für den Leiter KAEP ist deutschlandweit einheitlich geregelt.

\section{Frage 9}

Welche Aussage ist falsch? Der Alarm- und Einsatzplan zur Bewältigung einer „B-Lage“ ...

A sollte die Alarmierungswege definieren.

B sollte die Information von Angehörigen regeln.

C sollte die Raumordnung festlegen.

D sollte die gesamte Finanzierung regeln.

E sollte die Materialbevorratung regeln.

\section{Frage 10}

Welche Aussage ist falsch?

A Die KAEP sollte einen Plan zur Bewältigung eines Massenanfalls von Patienten beinhalten.

B Die Klinikeinsatzleitung setzt sich nur aus medizinischem Personal zusammen.

C Die gemeinsame Planung und Kommunikation mit der Feuerwehr, dem Rettungsdienst und den Vertretern des Katastrophenschutzes ist ein wichtiger Bestandteil der KAEP.

D Die Krankenhausleitung muss personelle Ressourcen für die KAEP zur Verfügung stellen.

E Die Expertengruppe für die KAEP besteht aus Vertretern diverser Berufsgruppen (Medizin, Technik, Logistik). 